\title{
Universal features of fluctuations
}

\author{
Robert Botet ${ }^{\dagger}$ and Marek Płoszajczak ${ }^{\ddagger}$ \\ † Laboratoire de Physique des Solides - CNRS, Bâtiment 510, Université Paris-Sud \\ Centre d'Orsay, F-91405 Orsay, France \\ and \\ ¥ Grand Accélérateur National d'Ions Lourds (GANIL), \\ CEA/DSM - CNRS/IN2P3, BP 5027, F-14076 Caen Cedex, France
}

(November 1, 2018)

\begin{abstract}
Universal scaling laws of fluctuations (the $\Delta$-scaling laws) can be derived for equilibrium and offequilibrium systems when combined with the finite-size scaling analysis. In any system in which the second-order critical behavior can be identified, the relation between order parameter, criticality and scaling law of fluctuations has been established and the relation between the scaling function and the critical exponents has been found.
\end{abstract}

\section{INTRODUCTION}

Recent years have witnessed an intense experimental and theoretical activity in search of scale-invariance and fractality in multihadron production processes [1]. The creation of soft hadrons in these processes is related to the strong-coupling, long-distance regime of Quantum Chromodynamics (QCD) whose exploration remains the sacred grail of the high-energy particle physics. Through a multitude of experimental studies of particle density fluctuations and particle correlations, new informations have been gathered which prompted the suggestion to investigate the pattern of multiplicity fluctuations in the small domains of phase-space looking for the scale-invariance and fractality [2]. New measurements has triggered revival of interest in the theory of fluctuations in complex systems.

In this work we shall discuss universal features of fluctuations of physical quantities in a $D$-dimensional, $N$ body system, where $N$ is essentially finite. We are particularly interested in the self-similar systems, such as the fractal objects or the thermodynamic systems at the second-order phase transition. By the self-similarity we mean that the characteristic length $\sim\left(N^{*}\right)^{1 / D}\left(N^{*}\right.$ is the characteristic size), which could be associated with the disappearance of fluctuations cannot be defined. The progress in this domain is associated with the development of the renormalization group methods. Actually, there is a close connection of the renormalization group ideas and the limit theorems in the probability theory. This basic idea stems from the works of Bleher, Sinai [3] and Jona-Lasinio [4], and consists of splitting the whole system into correlated blocks. Then the probability distribution of the investigated physical quantity is calculated for the blocks, and the renormalization procedure is expressing the distribution for large blocks in terms of the distributions for the smaller ones. These probability distributions dealing with larger and larger number of subunits are assumed to be given by probabilistic limit laws and the parameters of these distributions, properly renormalized at each step, are expected to tend to some finite limit. When this scheme is realized, the renormalization procedures gives the universal behavior of the infinite system.

Following this approach, we shall discuss how the universal scaling laws of fluctuations of different observables appear in finite systems. In particular, we shall consider the order parameter fluctuations in any equilibrium or non-equilibrium system in which the second-order critical behavior can be identified. These considerations will provide an understanding of the relation between the order parameter, the criticality and the scaling law of fluctuations. The notion of the relevant observable will also appear from this discussion.

\section{BROAD DISTRIBUTIONS AND LONG-RANGE CORRELATIONS}

\section{A. A basic example of the probabilistic limit laws}

Most physically interesting 'macroscopic variables' are defined as sum of many 'local random variables'. To understand such a 'macroscopic behavior' is to seek for some universal limit behavior. The simplest, non-trivial example is when the local variables are uncorrelated. Suppose that we make $N$ trials and a certain event has finite probability $p_{o}$ to occur at each trial. In this chain of trials, we are looking for the behavior of the probability distribution $P_{N}[m]$ to get exactly $m$ events during $N$ trials. In the following, $m$ will be called the multiplicity. This kind of process could describe for example the successive emission of particles : at each time interval $\tau$, one particle is emitted with a probability $p_{o}$ from a parent body. The equation for the probability distribution of the number of emitted particles in the time interval $n \tau$ :

$$
P_{N}[m]=\left(1-p_{o}\right) P_{N-1}[m]+p_{o} P_{N-1}[m-1] \text {, }
$$


tells that the multiplicity at stage $N$ is equal to $m$ if, either it was $m$ at stage $N-1$ and then the emission does not occur at the following trial, or it was $m-1$ at stage $N-1$ and the emission occurs in the last trial. In particular, eq. (11) shows that the average multiplicity is $<m>=N p_{o}$. When the number of trials $N$ becomes large, the probability distribution $P_{N}[m]$ tends to a normal law :

$$
\begin{gathered}
\lim _{<m>\rightarrow \infty}<m>^{1 / 2} P_{N}[m] \longrightarrow \\
\longrightarrow a_{p_{o}} \exp \left[-b_{p_{o}}\left(\frac{m-<m>}{<m>^{1 / 2}}\right)^{2}\right],
\end{gathered}
$$

with $b_{p_{o}}=1 /\left[2\left(1-p_{o}\right)\right]$. This form of the normal law expresses a relation between the scaled distribution $<m>^{1 / 2} P_{N}[m]$, and the shifted scaled multiplicity parameter $(m-<m>) /<m>^{1 / 2}$.

Let us now introduce the correlation between the two consecutive trials by prohibiting the successive emission. In this case, the probability distribution verifies :

$$
P_{N}[m]=(1-p) P_{N-1}[m]+p P_{N-2}[m-1] .
$$

It is a simple exercise to obtain the solution of eq. (3) : the leading order of the average multiplicity is $\langle m\rangle \sim$ $n p /(1+p)$ [5] and the limit distribution is given again by a normal law (2) with $b_{p_{o}}=1 /\left[2\left(1-p_{o}\right)\left(1-2 p_{o}\right)\right]$. Hence, the short-range correlations in (3) reduce the variance of the probability distribution by a factor $1-2 p_{o}$. It is important to realize a fundamental difference between the limit law, which is universal in the sense that most details of the considered process are unessential, and the parameters of the limit laws, which strongly depend on the specific features of the process.

\section{B. Central limit and stable laws for non-correlated variables}

Let us consider the additive, 'global' quantity : $Y_{N}=$ $\sum_{n=1}^{N} X_{n}$, expressed in terms of 'local' microscopic variables $X_{n}$. The fundamental problem in the statistical description of composed objects is to find a limit distribution of this variable, with $N$ being the number of subunits of the system. This problem can be approached in two steps : (i) when the random variables $X_{n}$ are all independent, then (ii) when they are correlated. The first step has been solved entirely [6]. The second step, when the variables are correlated, is not yet completely solved but some precise results and tools are available (see Ref. [7] for recent references).

In the following, we will work with the probability density : $f(x) d x=\operatorname{Prob}[x \leq X<x+d x]$, its characteristic function :

$$
\phi_{X}(k)=\int_{-\infty}^{\infty} e^{i k x} f(x) d x
$$

and the expansion of the characteristic function in a series of cumulants near $k=0$ :

$$
\begin{aligned}
\ln \phi_{X}(k) & =i k<X>- \\
& -\frac{k^{2}}{2}\left(<X^{2}>-<X>^{2}\right)+\ldots
\end{aligned}
$$

The importance of the characteristic function comes from the convolution theorem which tells that, if $X_{1}$ and $X_{2}$ are two independent random variables and $\phi_{X_{1}}(k)$ and $\phi_{X_{2}}(k)$ their characteristic functions, then the characteristic function of the random variable $Y=c_{1} X_{1}+c_{2} X_{2}$ is : $\phi_{Y}(k)=\phi_{X_{1}}\left(c_{1} k\right) \phi_{X_{2}}\left(c_{2} k\right)$ ( $c_{1}$ and $c_{2}$ are real numbers). In other words, the density of the sum of two independent variables is the product of the convolution of the two probability densities. The key point to determine all possible limit distribution of the random additive variable is the 'stability problem': What are all probability densities $f(x)$, such that, if $X_{1}$ and $X_{2}$ are two independent random variables of a probability density $f(x)$, and $c_{1}$ and $c_{2}$ are two real numbers, then $Y=c_{1} X_{1}+c_{2} X_{2}$ is a random variable with the same probability density $f(x)$ ? It turns out that the stable distributions are those for which the logarithm of their characteristic function is given by the formula :

$$
\ln \phi(k)=i \gamma k-c|k|^{\mu}\left(1+i \beta \frac{k}{|k|} \omega_{\mu}(k)\right),
$$

with four real parameters : $\gamma, c, \beta$ and $\mu$, where $c>0$, $|\beta| \leq 1$ and $0<\mu \leq 2$. In addition : $\omega_{\mu}(k)=\tan (\pi \mu / 2)$ if $\mu \neq 1$, else $\omega_{1}(k)=2 \ln (k / \pi)$. This four-parameter family of functions can be written in different forms. One of them is :

$$
\begin{aligned}
& P_{\mu, \beta}\left(c^{1 / \mu} x+\gamma\right)=\frac{1}{\pi c^{1 / \mu}} \times \\
\times & \operatorname{Re}\left[\int_{0}^{\infty} \exp \left(-i k x-\left(1+i \beta \tan \frac{\pi \mu}{2}\right) k^{\mu}\right) d k\right]
\end{aligned}
$$

if $\mu \neq 1$, and

$$
\begin{aligned}
& P_{1, \beta}(c x+\gamma)=\frac{1}{\pi c} \times \\
\times & \int_{0}^{\infty} \exp (-k) \cos \left(k\left(x+\frac{2 \beta}{\pi} \ln k\right)\right) d k
\end{aligned}
$$

if $\mu=1$. For $|x| \rightarrow \infty, P_{\mu, \beta} \propto|x|^{-(1+\mu)} \quad(0<\mu<2)$. For $0<\mu<1$ and $\beta \neq 0$, there is an essential singularity when $x \rightarrow 0: P_{\mu, \beta} \propto \exp \left[-\alpha(\mu, \beta) x^{\mu /(1-\mu)}\right]$. For other values, $P_{\mu, \beta}$ is finite at the origin.

The parameters appearing in these formulas are not equally important. $\gamma$ corresponds to a shift of the whole distribution, while $c$ is a scale factor. These two parameters are related to a translation and a dilatation of the distribution and can always be put respectively equal to 0 and 1 by a suitable choice of the coordinates. The parameter $\beta$ is related to the asymmetry of the distribution and 
$\beta$ vanishes for an even distribution. The most important parameter is the characteristic exponent $\mu$. It cannot be larger than 2 because $P_{\mu, \beta}$ should be non-negative, and is larger than 0 to ensure the convergence at the origin. The normal (gaussian) distribution corresponds to $\mu=2$, and this is the only case where the variance $\sigma_{X} \equiv<X^{2}>-<X>^{2}$ is finite. In fact, using (A) and the form of the stable distributions, one may show that:

$$
\begin{array}{r}
0<\mu \leq 1 \longrightarrow<X>=\infty \text { and } \sigma_{X}=\infty \\
1<\mu<2 \longrightarrow<X><\infty \text { and } \sigma_{X}=\infty \\
\mu=2 \longrightarrow<X><\infty \text { and } \sigma_{X}<\infty .
\end{array}
$$

\section{Asymptotically stable laws - the domains of partial attraction}

Let us recall that the stable distribution is invariant when added to itself. One would like to know what kind of distributions of the local random variable $X_{n}$ are leading to the stable distribution $P_{\mu, \beta}$ when $N \rightarrow \infty$. Let $X_{1}, X_{2}, \ldots, X_{N}$ denotes a sequence of independent numbers distributed according to a density $f(x)$, and let $Y_{N}$ be a random variable :

$$
Y_{N}=X_{1}+\ldots+X_{N} \quad \text { if } \quad 0<\mu<1
$$

and

$$
Y_{N}=X_{1}+\ldots+X_{N}-N<X>\quad
$$

if $1<\mu<2$. Let us define : $\mathcal{Y}_{N}=Y_{N} / B_{N}$, with the parameter $B_{N}$ chosen in such a way that the probability distribution of $\mathcal{Y}_{\mathcal{N}}$ converges to the stable distribution $P_{\mu, \beta}$ when $N$ tends to the infinity. When the distribution $f(x)$ yields the limit density $P_{\mu, \beta}$ for $\mathcal{Y}$, then $f$ is said to belong to the domain of attraction of $P_{\mu, \beta}$. The ensemble of the probability densities $f(x)$, such that :

$$
\begin{aligned}
& \lim _{x \rightarrow \infty} \frac{\int_{x}^{\infty} f(x) d x+\int_{-\infty}^{-x} f\left(x^{\prime}\right) d x^{\prime}}{\int_{a x}^{\infty} f(x) d x+\int_{-\infty}^{-a x} f\left(x^{\prime}\right) d x^{\prime}} \sim a^{\mu} \\
& \lim _{x \rightarrow \infty} \frac{\int_{-\infty}^{-x} f\left(x^{\prime}\right) d x^{\prime}}{\int_{x}^{\infty} f\left(x^{\prime}\right) d x^{\prime}}=\frac{1-\beta}{1+\beta},
\end{aligned}
$$

constitutes the entire domain of attraction of $P_{\mu, \beta}$. We see that only the tail of the distribution $f(x)$ is significant. If $f(x) \sim|x|^{-(1+\mu)} \quad(0<\mu<2)$ for $|x| \rightarrow \infty$, then $f(x)$ belongs to the domain of attraction of $P_{\mu, \beta}$, i.e. the reduced global variable $\mathcal{Y}_{\mathcal{N}}$ has a limiting stable distribution of index $\mu$ when $N \rightarrow \infty$. Moreover, all parameters $B_{N}, \gamma, c, \beta$ are known explicitly. In particular, $B_{N} \sim N^{1 / \mu}$.

\section{Stable distributions and the self-similarity}

The stochastic process $Y_{N}$ is called self-similar with the Hurst exponent $H$ if both $Y_{\lambda N}$ and $\lambda^{H} Y_{N}$ have the same distribution. If $Y_{N}$ is given by (6), (7), respectively, and the probability distribution $P\left[\mathcal{Y}_{\mathcal{N}}\right]$ converges to the stable law $P_{\mu, \beta}$, then the stochastic process $Y_{N}$ is selfsimilar with the Hurst exponent $H=1 / \mu$, and $\left\langle Y_{N}\right\rangle \sim$ $N^{1 / \mu}=N^{g} \cdot g$ is the anomalous dimension related to the extensive variable $Y_{N}$.

\section{E. Concept of the $\Delta$-scaling}

The case : $1<\mu<2$, corresponds to an infinite variance of microscopic variable $X$ with finite mean value $<X>$. Let $M_{N}=X_{1}+\ldots+X_{N}$. In this case :

$$
<M_{N}>\sim<X_{1}>+\ldots+<X_{N}>=N<X>\text {. }
$$

The preceding characterization of the attraction domains of the stable laws yields :

$$
\operatorname{Prob}\left[y \leq \frac{M_{N}-N<X>}{B_{N}}<y+d y\right] \sim P_{\mu, \beta}(y) d y
$$

with $B_{N} \sim<M_{N}>^{1 / \mu}$. Introducing the probability density $f_{M_{N}}(m)$ of the variable $M_{N}$, one obtains the limit distribution :

$$
<M_{N}>^{1 / \mu} f_{M_{N}}(m) \sim P_{\mu, \beta}\left(\frac{m-<M_{N}>}{<M_{N}>^{1 / \mu}}\right)
$$

, when the number $N$ of subunits $X_{n}$ tends to the infinity. Notice that $P_{\mu, \beta}$ appears now as the scaling function with the scaling index $\Delta=1 / \mu$, which can be extracted by plotting $<M_{N}>^{1 / \mu} f_{M_{N}}(m)$ against a scaling variable $\left(m-<M_{N}>\right) /<M_{N}>^{1 / \mu}$. In this representation, different curves for systems or stochastic processes with different number of subunits $N$ collapse into a single scaling curve which characterizes the stable law. The scaling law (8) will be called in the following the $\Delta$-scaling law. We shall return to this form later when discussing the second-order critical behavior in finite systems.

The case $0<\mu<1$ is more subtle, because $\left\langle M_{N}\right\rangle$ cannot be defined. In this case, a limiting behavior is expected to take a form :

$$
N^{1 / \mu} f_{M_{N}}(m) \sim P_{\mu, \beta}\left(\frac{m}{N^{1 / \mu}}\right) .
$$

There is no connection between $\left\langle M_{N}\right\rangle$ and $N$ in (9). Instead, one can show that the typical value of $M_{N}$ for large values of $N$ is : $M_{N}^{*} \simeq N^{1 / \mu}$, so the correct scaling is :

$$
M_{N}^{*} f_{M_{N}}(m) \sim P_{\mu, \beta}\left(\frac{m}{M_{N}^{*}}\right) .
$$


Notice that (10) resembles the limit when $\mu \rightarrow 1$ of the scaling law (8). This scaling form is not very useful because $M_{N}^{*}$ is not as precisely defined as the average value. It shows however, that it does not make sense to search for the scaling relations such as (8) with the scaling exponent $1 / \mu$ larger than 1 .

\section{ORDER PARAMETER FLUCTUATIONS}

Fluctuations of the order parameter are expected to have different properties at the critical point and outside of it. Far from the critical point, the correlations are short-ranged and the results of the previous chapter apply in this case. On the contrary, fluctuations of the order parameter close to the critical point are correlated throughout the whole system. Hence, the 'macroscopic' order parameter cannot be written as a simple sum of uncorrelated block variables, and its asymptotically stable distribution differs from those discussed in chapt. 2. In the next section we shall discuss some features of these distributions and show that they satisfy the $\Delta$-scaling law.

\section{A. Finite-size scaling argument in the thermodynamical systems}

In the thermodynamic limit, the free energy density of an equilibrium system close to the critical point scales as [8] :

$$
f\left(\lambda^{\beta} \eta, \lambda \epsilon\right) \sim \lambda^{2-\alpha} f(\eta, \epsilon)
$$

where $\alpha, \beta$ are the usual critical exponents, $\eta$ is the intensive order parameter and $\lambda$ is the scale parameter. $\epsilon$ is the control parameter which equals 0 at the critical point. Even though finite systems do not exhibit the critical behavior, nevertheless their properties resemble those of infinite systems if the correlation length $\xi$ is larger or comparable to the typical length $L$ of the system. In this case, one speaks about the pseudocritical point at a distance :

$$
\epsilon \sim c N^{-1 / \nu D}
$$

from the true critical point [9. $N$ is the size of the $D$ dimensional system and $c$ is some dimensionless constant which is negative, if a maximum of the finitesize susceptibility lies in the ordered phase, or positive, if this maximum is in the disordered phase. One can then derive the finite-size scaling of the total free energy $F(\eta, \epsilon, N)=N f(\eta, \epsilon)$ at the pseudocritical point :

$$
F_{c}(\eta, N) \sim f\left(\eta N^{\frac{\beta}{2-\alpha}}\right) .
$$

In deriving (12), we used the hyperscaling relation : $2-$ $\alpha=\nu D$. The canonical probability density of the order parameter $P_{N}[\eta]$ is given by :

$$
P_{N}[\eta]=Z_{N}^{-1} \exp \left[-\beta_{T} F(\eta, \epsilon, N)\right],
$$

where the coefficient $\beta_{T}(\equiv 1 / T)$ is independent of $\eta(T$ is the temperature of the system). Using eq. (13), one may calculate not only the most probable value of the order parameter, which is the solution to the equation $\partial P_{N}[\eta] / \partial \eta=0$, but also the average value of the order parameter and the partition function

$$
Z_{N} \sim N^{-\frac{\beta}{2-\alpha}} \sim<\eta>\sim \eta^{*}
$$

$\eta^{*}$ denotes the most probable value of the order parameter. $\langle\eta\rangle$ vanishes for large values of $N$, since both $\beta$ and $2-\alpha=2 \beta+\gamma$ are positive. The probability density $P_{N}[\eta]$ obeys the first-scaling law :

$$
<\eta>P_{N}[\eta]=\Phi(\eta /<\eta>) \equiv \Phi(z)
$$

with

$$
\Phi(z) \sim \exp \left(-\beta_{T} f(a z, c)\right)
$$

The logarithm of scaling function $\Phi(z)$ corresponds to the non-critical free energy density at the renormalized distance $\epsilon=c$ from the critical point. The scaling law (15), which is analogous to (10), can be rewritten as :

$$
<m>P_{N}[m]=\Phi\left(z_{(1)}\right)
$$

where $m=N \eta$ is the extensive order parameter and $z_{(1)}$ is :

$$
z_{(1)}=\left(m-m^{*}\right) /<m>
$$

$m^{*}$ denotes the most probable value of $m$. The scaling domain is defined by the asymptotic behavior of $P_{N}[m]$ when $m \rightarrow \infty$ and $\left\langle m>\rightarrow \infty\right.$, but $z_{(1)}$ has a finite value. Assuming that the scaling framework of the second-order phase transition holds, the scaling relation (15) is valid independently of the explicit reasons of changing $\langle m>$, and independently of any phenomenological details. In other words, an explicit relation between the size $N$ of the system and $\langle m\rangle$ need not to be known.

If the order parameter is related to the multiplicity of fragments or produced particles, like in the fragmentation - inactivation - binary (FIB) process [10], then the first scaling (15) becomes identical to the well known KNO-scaling [12]. Only in this case, the multiplicity of produced particles is the relevant observable. 


\section{B. Tail of the scaling function}

What are the properties of the scaling function for large values of $m /<m>$ ? The correct way of approaching this problem is to study the system subject to a small field $h$ which is conjugate to the order parameter and breaks the symmetry of the distribution. This consideration yields [13] :

$$
\Phi(z) \sim \exp \left(-a z_{(1)}^{\delta+1}\right) \equiv \exp \left(-a z_{(1)}^{\hat{\nu}}\right),
$$

with $\delta=(2-\alpha-\beta) / \beta$. The coefficient $a$ in (19) depends regularly on the temperature.

One can express this relation in a different way. The anomalous dimension for an extensive quantity $m=N \eta$ can be defined as :

$$
g=\lim _{N \rightarrow \infty} g_{N}=\lim _{N \rightarrow \infty} \frac{d}{d \ln N}(\ln <m>) .
$$

One can see from (14) and the Rushbrooke relation : $\alpha+2 \beta+\gamma=2$, that the anomalous dimension is :

$$
g=1-\beta /(\gamma+2 \beta) .
$$

Since $\alpha$ and $\beta$ are both positive, therefore $g$ is contained between $1 / 2$ and 1 for equilibrium systems at the critical point of the second-order phase transition. One may note also that :

$$
\hat{\nu}=\frac{1}{1-g}=\frac{2-\alpha}{\beta}>2 .
$$

Whenever the cluster-size can be correctly defined, like for example in the case of percolation or Ising models, the exponent $\tau$ of the power-law cluster-size distribution $n_{s} \sim s^{-\tau}$ satisfies :

$$
g=\frac{1}{\tau-1} \quad \text { and } \quad \hat{\nu}=\frac{\tau-1}{\tau-2} .
$$

The allowed values at the critical point are : $2<\tau<3$. Consequently, one can learn from the cluster-size distribution whether the equilibrium system is at the critical point and whether the considered extensive quantity can be identified with the order parameter. For this kind of equilibrium phase transitions, the size of the largest cluster is the order parameter since :

$$
<s_{\max }>\sim N^{\frac{1}{\tau-1}} \equiv N^{g} .
$$

The cluster multiplicity could be the order parameter if $\tau<2$, but this can be satisfied only for certain offequilibrium phase transitions, such as for example the shattering phase transition in the FIB process 10 or in the dissipative perturbative QCD [1].

\section{The $\Delta$-scaling law}

What happens if the observable $m$ is the $N$-dependent function of the order parameter :

$$
m=N^{a_{1}}-\eta \text { with } a_{1}>g .
$$

Writing (17) with $m$ instead of $\eta$ and taking into account that $P_{N}[\eta] d \eta=P_{N}[m] d m$, one finds :

$$
<m>^{\Delta} P_{N}[m]=\Phi\left(z_{(\Delta)}\right),
$$

with the scaling variable :

$$
z_{(\Delta)}=\left(m-m^{*}\right) /<m>^{\Delta},
$$

where :

$$
\Delta=g / a_{1}<1 .
$$

The normalization of $P_{N}[m]$ and the definition of $<m>$ provide the two constraints which are consistent with : $0<\Delta \leq 1$. This $\Delta$-scaling law $(0<\Delta<1)$ is satisfied, as shown in sect. 2.5 , by the asymptotically stable distributions with $1<\mu<2$ (eq. (8)). The asymptotically stable distributions $P_{\mu, \beta}$ with $0<\mu<1$ satisfy the first scaling law, i.e. the limiting case of the $\Delta$-scaling for $\Delta=1$.

The scaling function $\Phi\left(z_{(\Delta)}\right)$ has the identical form as $\Phi\left(z_{(1)}\right)$, except for the inversion of the abscissa axis. In particular, its tail for large $z_{(\Delta)}$ has the same form :

$$
\Phi\left(z_{(\Delta)}\right) \sim \exp \left(-z_{(\Delta)}^{\hat{\nu}}\right)=\exp \left(-z_{(\Delta)}^{\frac{1}{1-g}}\right)
$$

as given in (19). Notice that the $\Delta$-scaling of an extensive variable : $\hat{m}=N(1-\eta) \equiv N \hat{\eta}$, can be used to determine directly the anomalous dimension, since in this case : $\Delta=g$. At the phase transition : $\langle N \hat{\eta}\rangle \sim N$, but the finite-size corrections are algebraic. One should mention in passing that if $a_{1}<g$, then $\langle m>\sim N\langle\eta\rangle$ and $\Delta=1$.

It is important to realize that the $\Delta$-scaling $(0<\Delta<$ $1)$ and the first-scaling $(\Delta=1)$ laws are satisfied by the distributions of the quantities which are the sum of either independent or correlated random variables. In this sense, these scaling laws are the fundamental characteristics of the composed statistical system.

\section{Cumulant relations}

Let us now introduce the cumulants $\kappa_{q}$ from the formula for the moment expansion of the probability distribution $P[m]$ :

$$
\ln \left(\sum_{m=0}^{\infty} P[m] \exp (m u)\right)=\sum_{q=0}^{\infty} \frac{u^{q}}{q !} \kappa_{q} .
$$


In the case of $\Delta$-scaling law (eq. (25)), the cumulants satisfy :

$$
\kappa_{q} \sim<m>^{q \Delta} \equiv<m>^{q H} \quad(q \geq 2) .
$$

In the case of the first-scaling law (eq. (17)), one finds :

$$
\kappa_{q} \sim<m>^{q} .
$$

Notice also that the scaled variance $\kappa_{2} / \kappa_{1}^{2}$ is independent of $N$ for $\Delta=1$ and tends to 0 when $N \rightarrow \infty$ for $0<$ $\Delta<1$. In this sense, the scaling law (17) is the large fluctuation limit of the probability distribution $P_{N}[m]$.

\section{E. The intermittent behavior}

Intermittency in particle physics is defined usually as the scale-invariance of the factorial moments with respect to the change of the size of the phase-space cell $\delta y$ :

$$
F_{q}(\delta y) \propto(\delta y)^{-\phi_{q}},
$$

when $\delta y \rightarrow 0$. The exponent $\phi_{q}$ is called the intermittency index. For the multifractal distribution we have :

$$
\sum_{i} p_{i}^{q}(\delta y)=<p_{i}^{q-1}(\delta y)>\propto(\delta y)^{\tau(q)},
$$

where $p_{i}(\delta y)$ is the probability for the particle to be in the bin $i$ of size $\delta y$ and $\tau(q)$ is given by :

$$
\tau(q)=(q-1) D_{q} \Delta .
$$

Since :

$$
F_{q} \sim(\delta y)^{-(q-1) D}<p_{i}^{q-1}>\quad,
$$

where $D$ is the support dimension, therefore :

$$
\phi_{q}=(q-1)\left(D-D_{q} \Delta\right) .
$$

The quantity $D_{q} \Delta$ appears here as the apparent Renyi dimensions. Hence, knowing the intermittency indices and the value of $\Delta$, one can extract the 'true' multifractal spectrum.

\section{F. Scaling laws and the self-similarity}

The self-similarity of the statistical system which obeys the first-scaling law (eqs. (17), (18)) means that :

$$
<N \eta>_{\lambda N} \sim \lambda^{g}<N \eta>_{N} \equiv \lambda^{H}<N \eta>_{N} .
$$

The Hurst exponent is equal to the anomalous dimension $g$ (eq. (20)). In the case of the $\Delta$-scaling, $H \equiv g=$ $a_{1} \Delta$ (see eq. (27)). The exponent $a_{1}$ is the apparent anomalous dimension for $m$ defined by (24) : $<m>_{\lambda N} \sim$
$\lambda^{a_{1}}<m>_{N}$. It is easy to see, that these properties are analogous to those we have discussed in sect. 2.4 with $Y_{N}$ corresponding to $N \eta$ and $\sum X_{i}$ corresponding to $m$. Namely :

$$
a_{1}=1, \quad g=1 / \mu, \quad \Delta=1 / \mu
$$

for $1<\mu<2$, whereas

$$
a_{1} \equiv g=1 / \mu, \quad \Delta=1
$$

for $0<\mu<1$.

\section{G. The off-critical scaling}

Away from the critical region, if $m$ is the order parameter then the finite system exhibits the second-scaling law (the limit $\Delta=1 / 2$ of the $\Delta$-scaling law (25), (260) in the ordered phase, and the first-scaling law (eqs. (17, (18)) in the disordered phase. In both cases, the tail of the scaling function is gaussian $(\hat{\nu}=2)$ and the finite-size corrections are exponential. The second-scaling law has been found in the shattering phase of the non-equilibrium FIB process [14] and in the 'liquid' phase of the equilibrium percolation process [15. The first-scaling law with the gaussian tail has been found in the disordered phase of the Ising model [16].

One would like to know how a finite system evolves close to the critical point when the control parameter $\epsilon$ tends slowly to $0: \epsilon \sim N^{2 a_{1}-2}(\epsilon<1)$ [13]. In this case, $<m>$ and $m^{*}$ have a common behavior $\sim N^{a_{1}}$, and the probability distribution $P_{N}[m]$ satisfies the scaling :

$$
<m>^{\Delta} P_{N}[m] \sim \exp \left(-c \frac{\left(m-m^{*}\right)^{2}}{<m>^{2 \Delta}}\right)
$$

with $c>0$ and $\Delta=3 a_{1} / 2-1$. The case $a_{1}=1$, i.e., $\epsilon=$ const, corresponds to the second-scaling law. $a_{1}=3 / 4$ corresponds to the first-scaling law since the finite system is in the critical region $\left(a_{1}=g\right)$. In between these two limiting cases, the $\Delta$-scaling holds with $1 / 2<\Delta<1$. In all cases, the scaling function has a gaussian form.

\section{H. Equilibrium vs off-equilibrium critical systems}

Let us consider the bond percolation model. In the regular lattice, each site corresponds to a monomer and a proportion $p$ of active bonds between occupied sites is set randomly. Such a network results in a distribution of clusters which are defined as the ensemble of occupied sites connected by active bonds. For a critical value of active bonds $p_{c r}$, an 'infinite' cluster (the gel) spans the whole lattice. Infinite in this context means that the gel contains a finite fraction of the total mass of the system. The sol - gel phase transition can 
be suitably studied using moments of the number-sizedistribution $n_{s}: M_{k}^{\prime}=\sum_{s<s_{\max }} s^{k} n_{s}$, where the sum runs over all clusters with the exception of the largest cluster $s \equiv s_{\max }$ and the superscript ' recalls this constraint. The mass of the largest cluster is then : $N-M_{1}^{\prime}$. In the infinite system, one usually works with the normalized moments of the concentration-size-distribution $c_{s}$ : $m_{k}^{\prime}=\sum_{s<s_{\max }} s^{k} c_{s}$. The concentrations are normalized such that : $c_{s}=\lim _{N \rightarrow \infty} n_{s} / N$. The probability that a monomer belongs to the infinite cluster (the gel) is equal to $1-m_{1}^{\prime}$ with : $m_{k}^{\prime}=\lim _{N \rightarrow \infty} M_{k}^{\prime} / N$. Therefore, in the thermodynamic limit : $m_{1}^{\prime}=1$ for $p<p_{c r}$ and $m_{1}^{\prime}<1$ for $p>p_{c r}$, i.e., a finite fraction of the total number of sites belongs to the infinite cluster.

After this introduction, let us discuss the bondpercolation on the Bethe lattice with a coordination number $\hat{z}$ [17]. The concentration-size-distribution in this model is :

$$
c_{s}=\hat{z} \frac{((\hat{z}-1) s) !}{((\hat{z}-2) s+2) ! s !} p^{s-1}(1-p)^{(\hat{z}-2) s+\hat{z}}
$$

and the first normalized moment equals :

$$
m_{1}^{\prime}=\left(\frac{1-p}{1-p^{*}}\right)^{2 \hat{z}-2}
$$

with $p^{*}$ being the smallest solution of the equation :

$$
p^{*}\left(1-p^{*}\right)^{\hat{z}-2}=p(1-p)^{\hat{z}-2} .
$$

For $p<p_{c r}=(\hat{z}-1)^{-1}$, the only solution of eq. (39) is : $p^{*}=p$, but when $p$ is larger than $p_{c r}$, then there exists a smaller non-trivial solution which behaves as $p_{c r}-\left|p-p_{c r}\right|$ near $p_{c r}$. Above this threshold, the moment $m_{1}^{\prime}$ is smaller than 1 and behaves approximately as : $m_{1}^{\prime} \simeq 1-2(p-$ $\left.p_{c r}\right) /\left(1-p_{c r}\right)$.

For large values of the size $s$, the concentrations (38) can be rewritten using the Stirling approximation :

$$
c_{s} \sim s^{-5 / 2} \exp (-\alpha s) .
$$

$\alpha$ in (40) equals :

$$
\begin{aligned}
\alpha & =\ln \left[\frac{p}{p_{c r}}\left(\frac{1-p}{1-p_{c r}}\right)^{\hat{z}-2}\right] \\
& \simeq(\hat{z}-2) \ln (1-p)<0 .
\end{aligned}
$$

Hence :

$$
c_{s} \sim s^{-5 / 2}(1-p)^{-s(\hat{z}-2)} .
$$

At the percolation threshold, the concentrations follow the power-law and outside this threshold, an exponential cut-off is always present.

If we impose that $s_{\max }<\infty$, even in the thermodynamical limit, then the activation $p$ corresponding to $s_{\max }$ is smaller than $p_{c r}$ and the limiting activation $p_{0}$ equals $p_{c r}$. The limiting activation $p_{0}$ in percolation is analogous to the limiting temperature $T_{0}$ in the statistical bootstrap model (SBM) 18 . Both $p_{0}$ and $T_{0}$ follow from the consistency conditions in the two models and describe the asymptotic mass spectra. Obviously, the limiting value $p_{0} / T_{0}$ of the control parameter $p / T$ does not have to correspond to the critical point in the equilibrium system. For this to be true, the exponent $\tau$ of the mass spectrum must be contained in between 2 and 3.

One needs two critical exponents to describe completely the critical features. In percolation these are for example $\tau$ and $\sigma$ (the exponent of the mean cluster-size divergence). The $\Delta$-scaling analysis allows to determine only this exponent which is related to the anomalous dimension (e.g. $\tau$ in the percolation problem).

The insights gained from the numerical simulations of non-reversible aggregation equations (the Smoluchowski model) and FIB equations, both describing the offequilibrium phenomena, and the evidences from analytical solutions for gelling and non-gelling systems [13], provide strong indication that the scaling features, which were discussed in chapt. 2 for the distribution of the sum of random short-ranged correlated variables and in chapt. 3 for the distribution of the long-ranged correlated variables at critical point in equilibrium systems, are valid also for non-equilibrium systems. This universal behavior of equilibrium and non-equilibrium systems, has the deep foundation in the relation between renormalization group ideas for self-similar systems and the limit theorems of the probability theory for the $\Delta$-scaling laws, both for correlated and uncorrelated variables. The concept of statistical equilibrium does not intervene at this level.

\section{WHERE WE ARE, WHERE DO WE GO?}

There are two generic classes of dynamical critical phenomena, which are characterized by different 'relevant observables'. The first one is the sequential cluster fragmentation, where the average cluster size decreases and the cluster multiplicity increases during the process. The cluster (particle) multiplicity is an order parameter for this class of critical phenomena. The second one is the sequential cluster aggregation, where the average cluster size increases and the cluster multiplicity decreases during the process. The size of the largest cluster is here an order parameter. In the first class, one finds for example the shattering phase transition in the FIB process [10] as well as in the dissipative perturbative QCD process [11]. The second class contains for example the liquid-gas phase transition (Fisher model), the percolation transition or the sol-gel phase transition in the irreversible kinetic aggregation. Which of them is approximately realized in the multiparticle production process, 
can be discovered by studying the $\Delta$-scaling law, the tail of the scaling function, and the anomalous dimension, as described in the previous chapters.

\section{A. The perturbative QCD equations with the inactivation mechanism}

Let us call $P_{N}[n ; t]$ the probability to get a cluster multiplicity $n$ at time $t$, starting from an initial cluster of size $N$ at $t=0$. The time evolution equation for the multiplicity is given by the non-linear rate equation 10:

$$
\begin{aligned}
\frac{\partial G_{N}}{\partial t}(u, t) & =\sum_{j=1}^{N-1} \mathcal{F}_{j, N-j}\left[G_{j}(u, t) G_{N-j}(u, t)-\right. \\
& \left.-G_{N}(u, t)\right]+\mathcal{I}_{N}\left[1+u-G_{N}(u, t)\right]
\end{aligned}
$$

with the initial condition : $G_{1}(u, t)=1+u$, and the normalization condition : $G_{N}(u=0, t)=1 . G_{N}(u, t)$ is the generating function of the probability distribution : $G_{N}(u, t)=\sum_{n=1}^{\infty} P_{N}[n ; t](1+u)^{n}$. The partial derivative in (42) is taken at a fixed size $N$. The sum on the right hand side (rhs) of eq. (42) represents a binary fragmentation of the primary cluster $N$ into the daughter clusters of mass $j$ and $N-j$ respectively. The second term on the rhs side is the dissipative term which is responsible for the inactivation. One can transform the discrete variable $j$ in (42) into a continuous one $z=j / N$, which varies from 0 to 1 . With this change, the fragmentation kernel $\mathcal{F}_{j, N-j}$ is replaced by a splitting function : $\hat{\Phi}_{z, 1-z} \equiv N \mathcal{F}_{j, N-j}$.

In the limit of 'no dissipation', the FIB process yields the rate equations of gluodynamics in the Next to Next to Leading Logarithm Approximation (NNLLA) [19]. The inactivation term of FIB model yields a unique prescription of how to obtain the rate equations of perturbative QCD in the NNLLA, which would contain the hadronization effects phenomenologically through the inactivation mechanism of parton cascading.

The time $t$ in eq. (42) arises within the fragmentation and inactivation kernels, which themselves are probabilities per unit of $t$. We define then the time as : $t=T \ln Y$, where $T$ is a constant, $Y=\ln \left(N \Theta / Q_{0}\right), Q_{0}=$ const and $\Theta$ plays the role of time and orders the sequence of events. Assuming now that all physical quantities depend only on the variable $Y$ and not on $N$ and $\Theta$ separately, we transform (42) into :

$$
\begin{aligned}
\frac{\partial G}{\partial Y}(Y, u) & =\int_{0}^{1} \gamma_{0}^{2}(Y) \hat{\Phi}_{z, 1-z}[G(Y+\log z, u) \\
& \times G(Y+\log (1-z), u)-G(Y, u)] d z \\
& +\mathcal{R}(Y, u),
\end{aligned}
$$

with

$$
\mathcal{R}(Y, u)=\gamma_{0}^{2}(Y) \mathcal{I}(Y)[1+u-G(Y, u)],
$$

where : $\gamma_{0}^{2}(Y)=2 \pi^{-1} N_{C} \alpha_{s}(Y)=T / Y$, and $\alpha_{s}(Y)$ is the QCD running coupling constant. $\mathcal{I}(Y)$ in (44), is the inactivation function written in the new variables. It should be stressed that no cutoff is needed and the parton cascades are inactivated gradually by the inactivation function $\mathcal{I}(Y)$. The generalization of eqs. (43) to include both quarks and gluons can be found in 11 .

\section{B. Fragmentation scenario for $e^{+} e^{-}$collisions?}

Eqs. (43) of the dissipative gluodynamics have been applied for the description of the hadron multiplicity data in $e^{+} e^{-}$collisions [11]. The available data have been well described assuming the gaussian inactivation mechanism of the parton cascades. Based on that, it was concluded that the multihadron production in $e^{+} e^{-}$collisions resembles the critical off-equilibrium shattering process and the non-perturbative hadronization stage, approximated by the inactivation function $\mathcal{I}(Y)$, is slightly shifting this process from the critical line into the shattering phase. The small deviations from the first-scaling (the KNOscaling in this case) seen in the data for $\sqrt{s} \leq 100 \mathrm{GeV}$ is a finite-size effect related to the small size of an initial jet. The asymptotic region could not be precisely determined in these studies but, nevertheless, the limits imposed by the experimental data on the parameters of the inactivation function provide the upper limit for the borderline of an asymptotic region : $\sqrt{s} \sim 10^{2} \mathrm{TeV}$.

The experimental analysis of the ratio of the average charged multiplicity to the dispersion in $e^{+} e^{-} \rightarrow q \bar{q}$ events shows that the multiplicity distributions obey the first-scaling law 20. This confirms the relevance of the hadron multiplicity observable in this process. Unfortunately, the tail of the multiplicity probability distribution has not been resolved experimentally, so it is unknown at present whether $e^{+} e^{-} \rightarrow$ hadrons is the critical process at presently available energies and whether the hadron multiplicity is the order parameter in this process. Nevertheless, even though the experimental data are yet incomplete in many respects, the fact that such a simple observable as the hadron multiplicity is relevant allows to hope that the future studies at still higher collision energies will solve the enigma of possible off-equilibrium shattering phase transition [10] in the process : $e^{+} e^{-} \rightarrow$ hadrons. This specific transition, dominated by the perturbative regime of the QCD, is extremely interesting even though unrelated with the quark-gluon phase transition, which in turn is dominated by the strong-coupling regime of the QCD.

\section{Aggregation scenario for $p \bar{p}$ and $A A$ collisions?}

Hadron-hadron and nucleus-nucleus collisions show quite distinct features from those of lepton-lepton col- 
lisions. First of all, the multiplicity distributions show a clear deviation from the first-scaling law [21. The $p \bar{p}$ data for multiplicity distributions at $\sqrt{s}=200,546$ and $900 \mathrm{GeV}$ 21] can be approximately superposed at around the maximum in the variables of the $\Delta$-scaling law with $\Delta=0.922$. On the other hand, the strong scaling violations are seen in the tail for large multiplicities showing that the global scaling of multiplicity distributions for different $\sqrt{s}$ may not be possible. In this context, the two-component scenario including soft and semi-hard classes of events has been discussed [23]. The system size-dependence of these components cannot be accurately determined based on the existing data, so it is at present difficult to establish a physical meaning of this scenario. One should however mention, that finite systems in the neighborhood of the first-order liquid-gas phase transition, show a similar two-component behavior in the cluster multiplicity.

Correlation studies have disclosed the deficiency of the Lund Monte Carlo program PYTHIA containing jets, resonance production and the Bose-Einstein correlations as final state interactions, for the description of the differential density correlation function 24]. The correlations are both overestimated at high $p_{T}$ (high multiplicity) and underestimated at low $p_{T}$ (low multiplicity) by PYTHIA 24,25. Significant improvement can be gained assuming existence of clusters of low $p_{T}$ [24,26,27, as included in the non-diffractive event generator GENCL [26]. The picture of hadron-hadron collisions which emerges from these phenomenological studies shows the necessity of a combined description of hard scattering processes followed by the string fragmentation (the perturbative QCD regime), and the formation and decay of clusters in the non-perturbative QCD regime. The absence of an adequate computer program simulating these two aspects of hadron-hadron (nucleus-nucleus) collisions is presently a principle obstacle, slowing down a progress in the understanding of the production mechanism of soft hadrons in the strong-coupling, long-distance regime of QCD. The theoretical activity aiming at the development of such program should be accompanied by experimental studies of the appropriate methods to evidence the existence of clusters and to study their sizes.

The arguments put forward in favour of the cluster picture refer often to the SBM [18]. In this model, the number of species of possible constituents of the fireballs and the number of species of fireballs grow asymptotically like $\sim m^{-\tau} \exp \left(m / T_{0}\right)$, with $\tau=3$ [28]. As discussed in chapt. 3 , this value of $\tau$ excludes a possible identification of $T_{0}$ with the critical temperature of the thermodynamical quark-gluon phase transition. Another family of models refers to the spontaneous breakdown of the chiral symmetry of the QCD vacuum at a critical temperature. In the approximation $m_{u} \approx m_{d} \approx 0, m_{s} \rightarrow \infty$, the chiral QCD phase transition is of second order and the order parameter is given in terms of the sigma and pion field condensates [29]. In general, we get the guidance in designing appropriate models simulating the critical behavior in finite temperature QCD from the lattice QCD calculations (see e.g. 30). In all these models, the proper characterization of the soft phase of QCD should involve an understanding of features of the largest cluster (fireball) distribution.

To design the relevant experimental observable in $p \bar{p}$ and $A A$ collisions is by far the most difficult task. At the same time, this objective is the most important challenge of soft physics which if unsolved will jeopardies the analysis of different phases of hot hadronic and quarkgluon matter. The hadron (baryon) multiplicity distribution may contain useful information only if the studied system happens to be found at the critical point, what obviously limits the relevance of this observable. In this case, the the study of the scale dependence of correlations by means of the scale dependence of factorial (cumulant) moments of the density distribution, could reveal the underlying fractal structure of the largest cluster [31]. This is for the moment largely a theoretical possibility because the exact reconstruction of the geometry of the largest cluster may be even a harder experimental task than finding its mass event by event [32].

\section{CONCLUSIONS}

There is still a long way to understand the multihadron-production processes and, in particular, those aspects of these processes which are related to the strong-coupling long-distance regime of QCD. Experimental studies of correlations in small domains of the phase-space, posed the problem of the information contained in the multiparticle fluctuations of produced hadrons and, more generally, in the fluctuations of observables. We have shown that these fluctuations have universal features even in small finite systems, independently of whether the studied process is classical or quantum, equilibrium or non-equilibrium, dissipative etc. The information contained in the particle density and correlations can be extracted by determining (i) the scaling features (the $\Delta$-scaling law) of the measured probability distributions of the observable, in particular, the asymptotic properties of the scaling function, (ii) the anomalous dimension, and (iii) the scaling features of multiparticle correlations (clustering) in small phase-space cells. In this way, the relevance of the observable can be tested and/or the constraints on the multihadron-production mechanism can be determined.

Much of the experimental search for the scaling features in the multiparticle distributions has been motivated by the prediction of the KNO-scaling as an ultimate symmetry of the $S$-matrix in ultrarelativistic collisions. This scaling is however only a particular case of the general $\Delta$-scaling law which has been introduced only 
recently. For that reason, neither a systematic analysis of the deviation from the KNO-scaling in terms of the $\Delta$-scaling, nor the asymptotics of the scaling functions are available at present.

A crucial problem is the determination of the relevant observable for each multihadron-production process, which could exhibit different phases of the studied process through the non-trivial fluctuation pattern and its evolution. Whereas the hadron multiplicity distribution seems to be relevant for $e^{+} e^{-}$collisions, which share many aspects of the fragmentation scenario, this observable seems to be of secondary importance in $p \bar{p}$ and $A A$ collisions. In these latter collision processes, it is plausible that the aggregation scenario dominates and, hence, the cluster measures of the data have to be studied. The development of new methods of extraction of the 'biggest cluster' event by event, accompanied by the development of reliable programs simulating both the perturbative QCD regime and the clustering aspects in the non-perturbative regime, are the challenging, urgent problems which will determine the future evolution of the soft physics and the multiparticle correlations.

\section{Acknowledgements}

One of us (M.P.) wish to thank the organizers of TORINO 2000 for creating a lively and stimulating atmosphere during the meeting. We wish to thank also B. Buschbeck for stimulating discussions and for sending us her unpublished results.

[1] P. Bożek, M. Płoszajczak and R. Botet, Phys. Rep. 252 (1995) 101;

E.A. De Wolf, I.M. Dremin and W. Kittel, Phys. Rep. 270 (1996) 1.

[2] A. Białas and R. Peschanski, Nucl. Phys. B 273 (1986) 703.

[3] P.M. Bleher and Ja.G. Sinai, Comm. Math. Phys. 33 (1973) 23.

[4] G. Jona-Lasinio, Il Nuovo Cimento B 26 (1975) 99.

[5] For : $p=p_{o} /\left(1-p_{o}\right)$, one obtains the same average multiplicity $<m>=n p_{o}$ as in the uncorrelated case (eq. (11).

[6] P. Levi, Théorie de l'Addition des Variables Aléatoires, Gauthier, Paris, 1954.

[7] E. Eberlein and M.S. Taqqu (eds.), Dependence in Probability and Statistics : a Survey of Recent Results, Birkhauser, Boston, 1986.

[8] B. Widom, J. Chem. Phys. 43 (1965) 3898.

[9] M.E. Fisher and A.E. Ferdinand, Phys. Rev. Lett. 19 (1967) 169 .

[10] R. Botet and M. Płoszajczak, Int. J. of Mod. Phys. E 3 (1994) 1033.

[11] R. Botet and M. Płoszajczak, Zeitschrift f. Physik C 76
(1997) 257.

[12] Z. Koba, H.B. Nielsen, and P. Olesen, Nucl. Phys. B 40 (1972) 317.

[13] R. Botet and M. Płoszajczak, Phys. Rev. E 62 (2000) 1825.

[14] R. Botet and M. Płoszajczak, Phys. Rev. E 57 (1998) 7305.

[15] R. Botet, M. Płoszajczak, and V. Latora, Phys. Rev. Lett. 78 (1997) 4593.

[16] J-P. Bouchaud and A. Georges, Phys. Rep. 195 (1990) 127.

[17] M.E. Fisher and J.W. Essam, J. Math. Phys. 2 (1961) 609.

[18] R. Hagedorn, Suppl. Nuovo Cimento 3 (1965) 147; S. Frautschi, Phys. Rev. D 3 (1971) 2821.

[19] Yu.L. Dokshitzer et al., Basics of Perturbative QCD, Tran Thanh Van (ed.), Editions Frontieres, Gif-surYvette, 1991.

[20] P. Abreu et al., Phys. Lett. B 416 (1998) 233.

[21] G.J. Alner et al., Phys. Lett. B 160 (1985) 193; Phys. Rep. C 154 (1987) 247;

R.E. Ansorge et al., Zeitschrift f. Physik C 43 (1989) 357.

[22] R. Botet and M. Płoszajczak, in Proc. of XXVIII International Symposium on Multiparticle Dynamics, Delphi, Greece, N.G. Antoniou et al. (eds.), World Scientific, 1998.

[23] R. Ugoccioni and A. Giovannini, in Proc. of XXVIII International Symposium on Multiparticle Dynamics, Delphi, Greece, N.G. Antoniou et al. (eds.), World Scientific, 1998.

[24] B. Buschbeck, P. Lipa and F. Mandl, in Proc. of NATO Advanced Research Workshop on Hot Hadronic Matter : Theory and Experiment, Divonne - Les-Bains, France, Plenum, New York, 1994.

[25] C. Albajar et al., Nucl. Phys. B 345 (1990) 1.

[26] G.J. Alner et al., Nucl. Phys. B 291 (1987) 445.

[27] B. Buschbeck, private communication.

[28] W. Nahm, Nucl. Phys. B 45 (1972) 525.

[29] F. Wilczek, Int. J. Mod. Phys. A 7 (1992) 3911; K. Rajagopal and F. Wilczek, Nucl. Phys. B 399 (1993) 395.

[30] F. Karsch, in Proc. of Workshop on Nuclear Matter in Different Phases and Transitions, Les Houches, France, J-P. Blaizot, X. Campi and M. Płoszajczak (eds.), Kluwer Academic Publ., 1998.

[31] N.G. Antoniou, Y.F. Contoyiannis and F.K. Diakonos, in Proc. of XXVIII International Symposium on Multiparticle Dynamics, Delphi, Greece, N.G. Antoniou et al. (eds.), World Scientific, 1998;

N.G. Antoniou, in this volume.

[32] R.C. Hwa and Y. Wu, Phys. Rev. C 60 (1999) 054904. 